\title{
DECAY OF SOLUTION OF ANISOTROPIC DOUBLY NONLINEAR PARABOLIC EQUATION IN UNBOUNDED DOMAINS
}

\section{L.M. KOZHEVNIKOVA, A.A. LEONTIEV}

\begin{abstract}
This work is devoted to a class of parabolic equations with a double nonlinearity whose representative is a model equation

$$
\left(|u|^{k-2} u\right)_{t}=\sum_{\alpha=1}^{n}\left(\left|u_{x_{\alpha}}\right|^{p_{\alpha}-2} u_{x_{\alpha}}\right)_{x_{\alpha}}, \quad p_{n} \geq \ldots \geq p_{1}>k, \quad k \in(1,2) .
$$

For the solution of Dirichlet initial boundary value problem in a cylindrical domain $D=$ $(0, \infty) \times \Omega, \Omega \subset \mathbb{R}_{n}, n \geq 2$, with homogeneous Dirichlet boundary condition and compactly supported initial function, precise estimates the decay rate as $t \rightarrow \infty$ are established. Earlier these results were obtained by the authors for $k \geq 2$. The case $k \in(1,2)$ differs by the method of constructing Galerkin approximations that for an isotropic model equation was proposed by E.R. Andriyanova and F.Kh. Mukminov.
\end{abstract}

Keywords: anisotropic equation, doubly nonlinear parabolic equations, existence of strong solution, decay rate of solution.

\section{InTRODUCTION}

Let $\Omega$ be an unbounded domain in the space $\mathbb{R}_{n}=\left\{\mathbf{x}=\left(x_{1}, x_{2}, \ldots, x_{n}\right)\right\}, n \geq 2$. In the cylindrical domain $D=\{t>0\} \times \Omega$ we consider a Dirichlet initial boundary value problem for a second order anisotropic quasilinear parabolic equation

$$
\begin{gathered}
\left(|u|^{k-2} u\right)_{t}=\sum_{\alpha=1}^{n}\left(a_{\alpha}\left(u_{x_{\alpha}}^{2}\right) u_{x_{\alpha}}\right)_{x_{\alpha}}, \quad k \in(1,2), \quad(t, \mathbf{x}) \in D ; \\
\left.u(t, \mathbf{x})\right|_{S}=0, \quad S=\{t>0\} \times \partial \Omega ; \\
u(0, \mathbf{x})=\varphi(\mathbf{x}), \quad \varphi(\mathbf{x}) \in L_{k}(\Omega), \quad \varphi_{x_{\alpha}}(\mathbf{x}) \in L_{p_{\alpha}}(\Omega), \quad \alpha=\overline{1, n} .
\end{gathered}
$$

Nonnegative functions $a_{\alpha}(s), s \geq 0, \alpha=\overline{1, n}$ are assumed to obey the conditions $a_{\alpha}(0)=0$, $a_{\alpha}(s) \in C^{1}(0, \infty)$

$$
\begin{gathered}
\bar{a} s^{\left(p_{\alpha}-2\right) / 2} \leqslant a_{\alpha}(s) \leqslant \widehat{a} s^{\left(p_{\alpha}-2\right) / 2}, \\
\frac{p_{1}}{2} a_{\alpha}(s) \leqslant a_{\alpha}(s)+a_{\alpha}^{\prime}(s) s \leqslant \widehat{b} a_{\alpha}(s)
\end{gathered}
$$

with positive constants $\widehat{a} \geq \bar{a}, 2 \widehat{b} \geq p_{1}>k\left(p_{1} \leqslant p_{2} \leqslant \ldots \leqslant p_{n}\right)$. For example, $a_{\alpha}(s)=s^{\left(p_{\alpha}-2\right) / 2}$, $\alpha=\overline{1, n}, \widehat{b}=p_{n} / 2$.

L.M. Kozhevnikova, A.A. Leontiev, Decay of solution of anisotropic doubly nonlinear PaRABOLIC EQUATION IN UNBOUNDED DOMAINS.

(C) Kozhevnikova L.M., Leontiev A.A. 2013.

The work is supported by RFBR (grant no. 13-01-00081-a).

Submitted December 23, 2011. 
The work is devoted to studying the stabilization rate as $t \rightarrow \infty$ of the solution to problem (1)-(3) with a compactly supported initial function $\varphi(\mathbf{x})$.

The study of behavior of solutions to initial boundary value problems for linear and quasilinear parabolic equations of second and higher orders as $t \rightarrow \infty$ was done in the works of A.K. Gushchin, V.I. Ushakov, F.Kh. Mukminov, A.F. Tedeev, L.M. Kozhevnikova, R.Kh. Karimov, and others. The surveys of appropriate results can be found in [1], [2], [3].

In the isotropic case, i.e., as all $p_{\alpha}$ are same and equal to $p, p \geq 2$, for $k=2$ problem (1) -(3) was studied in work [3]. Estimates for the decay rate of the solution to a Cauchy problem for a parabolic degenerate equation with the anisotropic $p$-Laplacian and a double nonlinearity as $k \in(1,2)$ were established in the work of S.P. Degtyarev, A.F. Tedeev [4].

The questions on existence and uniqueness of solutions to an isotropic parabolic equation with a double nonlinearity were considered in the works by P.A. Raviart, J.L. Lions, A. Bamberger, O. Grange, F. Mignot, H.W. Alt, S. Luckhaus, F. Bernis, and others. However, to obtain a lower bound for the decay of the solution as $t \rightarrow \infty$ one needs additional smoothness.

F.Kh. Mukminov and E.R. Andriyanova [5] suggested a usual approach of construction a strong solution to a model isotropic parabolic equation with a double non-linearity in an unbounded domain on the basis of Galerkin approximations which in the cases $k \in(1,2)$ and $k \geq 2$ are constructed in different ways. In work [6] this method was adapted to a certain class of anisotropic parabolic equation like (1) as $k \geq 2$ and on the basis of Galerkin approximation they obtained an estimate for the admissible decay rate of the solution in an unbounded domain. The present work is the continuation of work [6] for the case $k \in(1,2)$.

We consider the domains located along a selected axis $O x_{s}, s \in \overline{1, n}$ (the domain $\Omega$ lies in the half-space $\mathbb{R}_{n}^{+}[s]=\left\{\mathbf{x} \in \mathbb{R}_{n} \mid x_{s}>0\right\}$, the cross-section $\gamma_{r}=\left\{\mathbf{x} \in \Omega \mid x_{s}=r\right\}$ is non-empty and bounded for each $r>0$ ). In what follows we shall employ the notation $\Omega_{a}^{b}=\left\{\mathbf{x} \in \Omega \mid a<x_{s}<b\right\}$, at that the values $a=0, b=\infty$ are omitted.

The initial function is assumed to be bounded and compactly supported so that

$$
\operatorname{supp} \varphi \subset \Omega^{R_{0}}, \quad R_{0}>0 .
$$

Theorem 1. Let the domain is located along the axis $O x_{s}, s \in \overline{1, n}$ and condition (6) is satisfied. Then there exist positive numbers $\kappa\left(p_{s}, k\right), \mathcal{M}\left(p_{s}, k\right)$ and a bounded solution $u(t, \mathbf{x})$ to problem (1)-(3) such that for all $t>0, r \geq 2 R_{0}$ the estimate

$$
\|u(t)\|_{L_{k}\left(\Omega_{r}\right)} \leqslant \mathcal{M} \exp \left(-\kappa\left[\frac{r^{p_{s}}}{t}\right]^{1 /\left(p_{s}-1\right)}\right)\|\varphi\|_{L_{k}(\Omega)}
$$

holds true.

On the basis of inequality (7) we establish a lower estimate for decay of a solution to problem (1) - (3) as $t \rightarrow \infty$.

The admissible stabilization rate for a solution to an high order isotropic quasilinear parabolic equation as $k=2$ was studied by A.F. Tedeev [7] for a Dirichlet initial boundary value problem and by N. Alikakos, R. Rostmanian [8] for a Cauchy problem.

Theorem 2. Suppose the domain is located along the axis $O x_{s}, s \in \overline{1, n}$ and condition (6) is satisfied. Then there exists a positive number $C\left(\varphi, k, p_{1}, \widehat{a}, \widehat{b}\right)$ and a bounded solution $u(t, \mathbf{x})$ to problem (1) -(3) such that for all $t \geq 0$ the inequality

$$
\|u(t)\|_{L_{k}(\Omega)} \geq\|\varphi\|_{L_{k}(\Omega)}(C(\varphi) t+1)^{-1 /\left(p_{1}-k\right)}
$$

holds true. 
We define a function

$$
\mu_{1}(r)=\inf \left\{\left\|g_{x_{1}}\right\|_{L_{p_{1}}\left(\Omega^{r}\right)} \mid g(\mathbf{x}) \in C_{0}^{\infty}(\Omega),\|g\|_{L_{k}\left(\Omega^{r}\right)}=1\right\}, \quad r>0 .
$$

we shall study the decay in the domain obeying the condition

$$
\lim _{r \rightarrow \infty} \mu_{1}(r)=0 \text {. }
$$

It is shown that if this condition is not satisfied, the maximal decay rate of a solution is attained, i.e., the estimate

$$
\|u(t)\|_{L_{k}(\Omega)} \leqslant M t^{-1 /\left(p_{1}-k\right)}, \quad t>0
$$

is valid (see [6, Cor. 2]).

We let

$$
\nu(r)=\inf \left\{\left\|g_{x_{1}}\right\|_{L_{p_{1}}\left(\gamma_{r}\right)} \mid g(\mathbf{x}) \in C_{0}^{\infty}(\Omega),\|g\|_{L_{p_{1}}\left(\gamma_{r}\right)}=1\right\}, \quad r>0 .
$$

We assume that the domain $\Omega$ satisfies the condition

$$
\int_{1}^{\infty} \nu^{p_{1} / p_{s}}(r) d r=\infty
$$

Let $r(t)$ be an arbitrary positive function obeying the inequality

$$
\left(\mu_{1}^{p_{1}}(r(t)) t\right)^{-1 /\left(p_{1}-k\right)} \exp \left(\kappa \int_{1}^{r(t)} \nu^{p_{1} / p_{s}}(\rho) d \rho\right) \geq 1, \quad t>0 .
$$

The existence of such function follows from (10). Moreover, it follows from (14), (10) that

$$
\lim _{t \rightarrow \infty} r(t)=\infty
$$

Theorem 3. Suppose the domain is located along the axis $O x_{s}, s \in \overline{2, n}$ and the conditions (6), (10), (13) are satisfied. Then there exist a positive number $M\left(p_{s}, p_{1},\|\varphi\|_{L_{k}(\Omega)}\right)$ and a bounded solution $u(t, \mathbf{x})$ to problem (1)-(3) such that the estimate

$$
\|u(t)\|_{L_{k}(\Omega)} \leqslant M\left(t \mu_{1}^{p_{1}}(r(t))\right)^{-1 /\left(p_{1}-k\right)}, \quad t>0,
$$

holds true.

If the conditions

$$
\begin{gathered}
\mu_{1}(r) \geq C r^{-a}, \quad r>1, \quad a, C>0, \\
\lim _{r \rightarrow \infty} \frac{1}{\ln r} \int_{1}^{r} \nu^{p_{1} / p_{s}}(\rho) d \rho=\infty
\end{gathered}
$$

are satisfied, one can let

$$
r(t)=t^{\varepsilon /\left(a p_{1}\right)}, \quad t>0, \quad \varepsilon \in(0,1),
$$

and estimate (15) casts into the form

$$
\|u(t)\|_{L_{k}(\Omega)} \leqslant M t^{-(1-\varepsilon) /\left(p_{1}-k\right)}, \quad t>0 .
$$

The choice of the function $r(t)$ by formula (16) is satisfactory since estimate (17) has an exponent close to the exponent $1 /\left(p_{1}-k\right)$ of lower bound (8). Other examples of the domains of revolution are provided in work [6]. 


\section{Auxiliary Statements}

Let $\|\cdot\|_{p, Q}$ be the norm in $L_{p}(Q), p \geq 1,(\cdot, \cdot)_{Q}$ be the scalar product in $L_{2}(Q)$ and the values $p=2, Q=\Omega$ are omitted. By $D_{a}^{b}=(a, b) \times \Omega$ we denote the cylinder, the values $a=0$ and $b=\infty$ can be absent.

A Banach space $\stackrel{\circ}{W}_{k, \mathbf{p}}^{1}(\Omega)$ is introduced as the completion of the space $C_{0}^{\infty}(\Omega)$ w.r.t. the norm

$$
\|u\|_{W_{k, \mathbf{p}}^{1}(\Omega)}=\sum_{\alpha=1}^{n}\left\|u_{x_{\alpha}}\right\|_{p_{\alpha}}+\|u\|_{k} .
$$

We introduce Banach spaces $\stackrel{\circ}{W} \underset{k, \mathbf{p}}{0,1}\left(D^{T}\right), \stackrel{\circ}{W} \underset{k, \mathbf{p}}{1,1}\left(D^{T}\right)$ as the completions of the space $C_{0}^{\infty}\left(D_{-1}^{T+1}\right)$ w.r.t. the norms

$$
\begin{gathered}
\|u\|_{W_{k, \mathbf{p}}^{0,1}\left(D^{T}\right)}=\|u\|_{k, D^{T}}+\sum_{\alpha=1}^{n}\left\|u_{x_{\alpha}}\right\|_{p_{\alpha}, D^{T}}, \\
\|u\|_{W_{k, \mathbf{p}}^{1,1}\left(D^{T}\right)}=\|u\|_{k, D^{T}}+\left\|u_{t}\right\|_{k, D^{T}}+\sum_{\alpha=1}^{n}\left\|u_{x_{\alpha}}\right\|_{p_{\alpha}, D^{T}},
\end{gathered}
$$

respectively.

Definition 1. By a generalized solution to problem (1)-(3), we call a function $u(t, \mathbf{x})$ such that for all $T>0 u(t, \mathbf{x}) \in \stackrel{\circ}{W}_{k, \mathbf{p}}^{0,1}\left(D^{T}\right)$ and the integral identity

$$
\int_{D^{T}}\left(-|u|^{k-2} u v_{t}+\sum_{\alpha=1}^{n} a_{\alpha}\left(u_{x_{\alpha}}^{2}\right) u_{x_{\alpha}} v_{x_{\alpha}}\right) d \mathbf{x} d t=\int_{\Omega}|\varphi(\mathbf{x})|^{k-2} \varphi(\mathbf{x}) v(0, \mathbf{x}) d \mathbf{x}
$$

is satisfied for each function $v(t, \mathbf{x}) \in \stackrel{\circ}{W} \underset{k, 1}{1,1}\left(D^{T}\right), v(T, \mathbf{x})=0$.

Conditions (5) imply inequalities

$$
\left(p_{1}-1\right) a_{\alpha}(s) \leqslant a_{\alpha}(s)+2 a_{\alpha}^{\prime}(s) s \leqslant \widehat{c} a_{\alpha}(s), \quad \widehat{c}=2 \widehat{b}-1, \quad s \geq 0, \quad \alpha=\overline{1, n},
$$

which can be rewritten as

$$
0 \leqslant\left(a_{\alpha}\left(z^{2}\right) z\right)^{\prime} \leqslant \widehat{c} a_{\alpha}\left(z^{2}\right), \quad z \in \mathbb{R}, \quad \alpha=\overline{1, n} .
$$

We let $A_{\alpha}(s)=\int_{0}^{s} a_{\alpha}(\tau) d \tau$, then, employing conditions 5 , we deduce the inequalities

$$
\frac{p_{1}}{2} A_{\alpha}(s) \leqslant a_{\alpha}(s) s \leqslant \widehat{b} A_{\alpha}(s), \quad s \geq 0, \quad \alpha=\overline{1, n} .
$$

Lemma 1. Each bounded set of reflexive Banach space is weakly compact (see [9, Ch. V, Sec. 19.7, Th. 1]).

Remark 1. The spaces $\stackrel{\circ}{W}_{k, \mathbf{p}}^{1}(\Omega), \stackrel{\circ}{W} \underset{k, \mathbf{p}}{0,1}\left(D^{T}\right)$ are reflexive separable Banach spaces (cf. [6, Rem. 1]).

Remark 2. In what follows, in order to avoid bulking while arguing, instead of the statements like "in a sequence $u^{M}$ one can select a subsequence $u^{M_{i}}$ converging in $L_{2}(\Omega)$ as $i \rightarrow \infty$ " we shall say shortly "a sequence $u^{M}$ selectively converges in $L_{2}(\Omega)$ as $M \rightarrow \infty$ ". In a similar way we shall employ the notion "selectively weakly converges", etc.

Lemma 2. $\operatorname{Let}^{M}(t, \mathbf{x}), M=\overline{1, \infty}, g(t, \mathbf{x})$ be functions in $L_{p}(Q), 1<p<\infty$ such that

$$
\left\|g^{M}\right\|_{p, Q} \leqslant C, \quad g^{M} \rightarrow g \text { as } M \rightarrow \infty \text { a.e. in } Q,
$$

then $g^{M} \rightarrow g$ as $M \rightarrow \infty$ weakly in $L_{p}(Q)$ (see [10, Ch. I, Sec. 1.4, Lem. 1.3]) 
Remark 3. Lemma 2 is formulated in [10] for a bounded domain $Q$, but it is true also for an arbitrary unbounded domain. We shall apply Lemma 2 for $Q=\Omega$ and for $Q=(0, T) \times \Omega$.

Lemma 3. Let the system of functions $\psi_{i}(\mathbf{x}) \in C_{0}^{\infty}(\Omega), i=\overline{1, \infty}$, is linearly independent and its linear span is a dense set in the space $\stackrel{\circ}{W}_{k, \mathbf{p}}^{1}(\Omega)$. By $P_{L}$ we denote the set of the functions $\sum_{i=1}^{L} d_{i}(t) \psi_{i}(\mathbf{x})$, where $d_{i}(t) \in C^{\infty}[0, T]$. Then the set $P=\bigcup_{L=1}^{\infty} P_{L}$ is dense in the space $\stackrel{\circ}{W}_{k, \mathbf{p}}^{1,1}\left(D^{T}\right)$.

Proof. Let us prove the density of the set $P$ in the space $C_{0}^{\infty}\left(D_{-1}^{T+1}\right)$. Let $v(t, \mathbf{x}) \in C_{0}^{\infty}\left(D_{-1}^{T+1}\right)$, obviously, $v(t, \mathbf{x}) \in C([-1, T+1] \rightarrow \stackrel{\circ}{W} \stackrel{1}{k}, \mathbf{p}(\Omega))$. We choose an arbitrary $\varepsilon$ and fix $\delta$ such that for all $t, t^{*} \in[-1, T+1]$ obeying $\left|t-t^{*}\right|<2 \delta$ the inequality

$$
\left\|v(t)-v\left(t^{*}\right)\right\|_{\stackrel{\circ}{W}_{k, \mathbf{p}}^{1}(\Omega)}<\varepsilon
$$

holds true.

We choose a finite sequence of points $t_{j}, j=\overline{1, N}$ such that $(-1, T+1)=\bigcup_{j=1}^{N}\left(t_{j}-\delta, t_{j}+\delta\right)$ and a partition of the unity

$$
\sum_{j=1}^{N} w_{j}(t)=1, \quad w_{j}(t) \in C_{0}^{\infty}\left(\left(t_{j}-\delta, t_{j}+\delta\right)\right) \quad 0 \leqslant w_{j}(t) \leqslant 1 .
$$

The definition of the system of the functions $\psi_{k}(\mathbf{x})$ implies that for each $j, j=\overline{1, N}$, there exists an index $L_{j}(\varepsilon)$ and numbers $f_{j k}$ such that

$$
\left\|v\left(t_{j}, \mathbf{x}\right)-\sum_{k=1}^{L_{j}} f_{j k} \psi_{k}(\mathbf{x})\right\|_{\stackrel{\circ}{k}, \mathbf{p}^{1}(\Omega)}<\varepsilon, \quad j=\overline{1, N}
$$

Let us show that the functions $\sum_{j=1}^{N} \sum_{k=1}^{L_{j}} w_{j}(t) f_{j k} \psi_{k}(\mathbf{x})=\sum_{k=1}^{L}\left(\sum_{j=1}^{N} w_{j}(t) f_{j k}\right) \psi_{k}(\mathbf{x}), L=\max _{j=1, N} L_{j}$, $f_{j k}=0, k>L_{j}$, approximate the function $v(t, \mathbf{x})$ in the norm of the space $\stackrel{\circ}{W_{k, \mathbf{p}}^{1}}(\Omega)$ uniformly in $t \in[-1, T+1]$. Indeed, employing (22), (23), we deduce

$$
\begin{aligned}
& \max _{t \in[-1, T+1]}\left\|v(t, \mathbf{x})-\sum_{j=1}^{N} \sum_{k=1}^{L_{j}} w_{j}(t) f_{j k} \psi_{k}(\mathbf{x})\right\|_{\stackrel{\circ}{k, p}_{k}^{1}(\Omega)}= \\
& =\max _{t \in[-1, T+1]}\left\|\sum_{j=1}^{N} w_{j}(t) v(t, \mathbf{x})-\sum_{j=1}^{N} \sum_{k=1}^{L_{j}} w_{j}(t) f_{j k} \psi_{k}(\mathbf{x})\right\|_{\stackrel{\circ}{W}, \mathbf{p}_{k}^{1}(\Omega)} \leqslant \\
& \leqslant \sum_{j=1}^{N} \max _{\left[-\delta+t_{j}, \delta+t_{j}\right]}\left\|w_{j}(t) v(t, \mathbf{x})-w_{j}(t) \sum_{k=1}^{L} f_{j k} \psi_{k}(\mathbf{x})\right\|_{\stackrel{\circ}{k}, \mathbf{p}^{1}(\Omega)} \leqslant \\
& \leqslant \sum_{j=1}^{N} \max _{\left[-\delta+t_{j}, \delta+t_{j}\right]}\left\|v(t, \mathbf{x})-\sum_{k=1}^{L} f_{j k} \psi_{k}(\mathbf{x})\right\|_{\stackrel{\circ}{k}, \mathbf{p}^{1}(\Omega)} \leqslant \\
& \leqslant \sum_{j=1}^{N} \max _{\left[-\delta+t_{j}, \delta+t_{j}\right]}\left\|v(t, \mathbf{x})-v\left(t_{j}, \mathbf{x}\right)\right\|_{\stackrel{\circ}{k}, \mathbf{p}^{1}(\Omega)}+ \\
& +\sum_{j=1}^{N}\left\|v\left(t_{j}, \mathbf{x}\right)-\sum_{k=1}^{L} f_{j k} \psi_{k}(\mathbf{x})\right\|_{\stackrel{\circ}{k}, \mathbf{p}^{1}(\Omega)} \leqslant N \varepsilon+N \varepsilon=2 N \varepsilon=\varepsilon_{1} .
\end{aligned}
$$


We introduce the notation $f_{k}(t)=\sum_{k=1}^{L} w_{j}(t) f_{j k}$. We take $w \in C_{0}^{\infty}\left(D_{-1}^{T+1}\right)$ and let $v(t, \mathbf{x})=w_{t}(t, \mathbf{x}) \in C_{0}^{\infty}\left(D_{-1}^{T+1}\right)$. According to proven above, for each $\varepsilon_{1}>0$ there exists $L\left(\varepsilon_{1}\right)$ such that

$$
\max _{t \in[-1, T+1]}\left\|w_{t}-\sum_{k=1}^{L\left(\varepsilon_{1}\right)} f_{k}(t) \psi_{k}(\mathbf{x})\right\|_{\stackrel{\circ}{k, \mathbf{p}}^{1}(\Omega)}<\varepsilon_{1}
$$

Consider the function $w(t, \mathbf{x})=\int_{-1}^{t} w_{\tau}(\tau, \mathbf{x}) d \tau$ and let us show that the functions $\sum_{k=1}^{L}\left(\int_{-1}^{t} f_{k}(\tau) d \tau\right) \psi_{k}(\mathbf{x})$ approximate the function $w(t, \mathbf{x})$ in the space $\stackrel{\circ}{W_{k, \mathbf{p}}^{1}} \underset{1}{1}(\Omega)$ uniformly in $t \in[-1, T+1]$. Indeed, as $L \rightarrow \infty$

$$
\begin{aligned}
& \max _{t \in[-1, T+1]}\left\|w(t, \mathbf{x})-\sum_{k=1}^{L} \int_{-1}^{t} f_{k}(\tau) \psi_{k}(\mathbf{x}) d \tau\right\|_{\stackrel{\circ}{k}_{k, \mathbf{p}}^{1}(\Omega)}= \\
& =\max _{t \in[-1, T+1]}\left\|\int_{-1}^{t} w_{\tau}(\tau, \mathbf{x}) d \tau-\sum_{k=1}^{L} \int_{-1}^{t} f_{k}(\tau) \psi_{k}(\mathbf{x}) d \tau\right\|_{W_{k, \mathbf{p}}(\Omega)}= \\
& =\max _{t \in[-1, T+1]}\left\|\int_{-1}^{t}\left(w_{\tau}(\tau, \mathbf{x})-\sum_{k=1}^{L} f_{k}(\tau) \psi_{k}(\mathbf{x})\right) d \tau\right\|_{\stackrel{\circ}{W}, \mathbf{p}_{k}^{1}(\Omega)} \leqslant \\
& \leqslant(T+2) \max _{\tau \in[-1, T+1]}\left\|w_{\tau}(\tau, \mathbf{x})-\sum_{k=1}^{L} f_{k}(\tau) \psi_{k}(\mathbf{x})\right\|_{\stackrel{\circ}{k}, \mathbf{p}^{1}(\Omega)} \rightarrow 0 .
\end{aligned}
$$

Denote $d_{k}(\tau)=\int_{-1}^{\tau} f_{k}(\rho) d \rho$, then inequality 24 and the latter relations as $L \rightarrow \infty$ imply

$$
\begin{aligned}
& \max _{\tau \in[-1, T+1]}\left\|w_{\tau}(\tau, \mathbf{x})-\sum_{k=1}^{L} d_{k}^{\prime}(\tau) \psi_{k}(\mathbf{x})\right\|_{\stackrel{\circ}{k}, \mathbf{p}_{\mathbf{p}}(\Omega)} \rightarrow 0 \\
& \max _{[-1, T+1]}\left\|w(\tau, \mathbf{x})-\sum_{k=1}^{L} d_{k}(\tau) \psi_{k}(\mathbf{x})\right\|_{W_{k, \mathbf{p}}^{\circ}(\Omega)} \rightarrow 0,
\end{aligned}
$$

that yields $\left\|w(\tau, \mathbf{x})-\sum_{k=1}^{L} d_{k}(\tau) \psi_{k}(\mathbf{x})\right\|_{\stackrel{\circ}{W}_{k, \mathbf{p}}^{1,1}\left(D_{-1}^{T+1}\right)} \rightarrow 0$.

Theorem 4. Let $\varphi(\mathbf{x}) \in \stackrel{\circ}{W_{k}}, \mathbf{p}(\Omega), p_{1} \geq k, k \in(1,2)$, then there exists a generalized solution $u(t, \mathbf{x})$ to problem (1)-(3) which for each $T>0$ satisfies the conditions

$$
\begin{gathered}
u \in L_{\infty}\left((0, \infty), \stackrel{\circ}{W}_{k, \mathbf{p}}^{1}(\Omega)\right) ; \\
|u|^{(k-2) / 2} u_{t} \in L_{2}\left(D^{T}\right), \quad u \in C\left([0, \infty), L_{k}(\Omega)\right) ; \\
u_{t} \in L_{k}\left(D^{T}\right) .
\end{gathered}
$$

At that, the inequalities

$$
(k-1)\|u(t)\|_{k}^{k}+k \bar{a} \sum_{\alpha=1}^{n} \int_{0}^{t}\left\|u_{x_{\alpha}}(\tau)\right\|_{p_{\alpha}}^{p_{\alpha}} d \tau \leqslant(k-1)\|\varphi\|_{k}^{k}, \quad t \geq 0,
$$




$$
(k-1) \frac{d}{d t}\|u(t)\|_{k}^{k}+k \bar{a} \sum_{\alpha=1}^{n}\left\|u_{x_{\alpha}}(t)\right\|_{p_{\alpha}}^{p_{\alpha}} \leqslant 0, \quad t>0
$$

hold true.

Proof. We choose a linearly independent system of functions $\psi_{i}(\mathbf{x}) \in C_{0}^{\infty}(\Omega), i=\overline{1, \infty}$, such that its linear span is a dense set in the space $\stackrel{\circ}{W}_{k, \mathbf{p}}^{1}(\Omega)$. This system is assumed to be orthonormalized in $L_{2}(\Omega)$. We let $I^{M}=\bigcup_{i=1}^{M} \operatorname{supp} \psi_{i}(\mathbf{x}), m_{i}=\max _{\mathbf{x} \in I^{M}}\left|\psi_{i}(\mathbf{x})\right|$.

We seek approximate solutions $u^{M}(t, \mathbf{x})$ as $u^{M}(t, \mathbf{x})=\sum_{i=1}^{M} c_{i}^{M}(t) \psi_{i}(\mathbf{x}), \quad M=\overline{1, \infty}$. At that, the functions $c_{i}^{M}(t), t \in[0, \infty)$, are determined by the system of ordinary differential equations

$$
\begin{gathered}
\left(\left(\left(\omega^{M}\right)^{k / 2-1} u^{M}\right)_{t}, \psi_{j}\right)+\sum_{\alpha=1}^{n}\left(a_{\alpha}\left(\left(u_{x_{\alpha}}^{M}\right)^{2}\right) u_{x_{\alpha}}^{M},\left(\psi_{j}\right)_{x_{\alpha}}\right)=0, \\
\omega^{M}=\left(u^{M}\right)^{2}+\frac{k}{2} \varepsilon^{M}, \quad j=\overline{1, M},
\end{gathered}
$$

(we shall choose the numbers $\varepsilon^{M}>0$ later) and by initial conditions

$$
c_{i}^{M}(0)=c_{i}^{M}, \quad i=\overline{1, M},
$$

chosen so that

$$
u^{M}(0, \mathbf{x})=\sum_{i=1}^{M} c_{i}^{M} \psi_{i}(\mathbf{x}) \rightarrow \varphi(\mathbf{x}) \text { in } \stackrel{\circ}{W} \underset{k, \mathbf{p}}{1}(\Omega) \text { as } M \rightarrow \infty .
$$

It implies immediately that

$$
\left\|u^{M}(0)\right\|_{W_{k, \mathbf{p}}^{1}(\Omega)} \leqslant E_{1}\left(\|\varphi\|_{W_{k, \mathbf{p}}^{1}(\Omega)}\right), \quad M=\overline{1, \infty} .
$$

Let us make sure that equations 30 are solvable w.r.t. the derivatives $\frac{d}{d t} c_{i}^{M}(t)$. It is obvious that equations 30 read as

$$
\begin{gathered}
\sum_{i=1}^{M} A_{j i}\left(c_{1}^{M}(t), \ldots, c_{M}^{M}(t)\right) \frac{d}{d t} c_{i}^{M}(t)=F_{j}\left(c_{1}^{M}(t), \ldots, c_{M}^{M}(t)\right), \quad j=\overline{1, M} \\
A_{j i}\left(c_{1}, \ldots, c_{M}\right)=\left(\left(\varepsilon^{M} \frac{k}{2}+(k-1)\left(\sum_{l=1}^{M} c_{l} \psi_{l}\right)^{2}\right)\left(\omega^{M}\right)^{k / 2-2} \psi_{i}, \psi_{j}\right)= \\
=\left(\psi_{i}, \psi_{j}\right)_{M}, i, j=\overline{1, M}, \quad F_{j}\left(c_{1}, \ldots, c_{M}\right)= \\
=-\sum_{\alpha=1}^{n} \sum_{i=1}^{M} c_{i}\left(a_{\alpha}\left(\left(\sum_{l=1}^{M} c_{l}\left(\psi_{l}\right)_{x_{\alpha}}\right)^{2}\right)\left(\psi_{i}\right)_{x_{\alpha}},\left(\psi_{j}\right)_{x_{\alpha}}\right), \quad j=\overline{1, M} .
\end{gathered}
$$

It is easy to check that $(g, h)_{M}, g, h \in C_{0}^{\infty}(\Omega)$, is a scalar product. Therefore, for each $t$ the matrix of the coefficients $A_{j i}\left(c_{1}^{M}(t), \ldots, c_{M}^{M}(t)\right)$ is the Gram matrix of the system of linearly independent vectors $\psi_{i}, i=\overline{1, M}$, and is invertible. This is why system (34) can be rewritten as

$$
\frac{d}{d t} c_{i}^{M}(t)=\sum_{j=1}^{M} A_{i j}^{-1}\left(c_{1}^{M}(t), \ldots, c_{M}^{M}(t)\right) F_{j}\left(c_{1}^{M}(t), \ldots, c_{M}^{M}(t)\right), \quad i=\overline{1, M}
$$


Let us establish the estimate for Galerkin approximations. We multiply $j$ th equation in (30) by $c_{j}^{M}(t)$ and sum up then all the equations in $j$ from 1 to $M$ that results in the identities

$$
\left(\left(\left(\omega^{M}\right)^{k / 2-1} u^{M}\right)_{t}, u^{M}\right)+\sum_{\alpha=1}^{n}\left(a_{\alpha}\left(\left(u_{x_{\alpha}}^{M}\right)^{2}\right) u_{x_{\alpha}}^{M}, u_{x_{\alpha}}^{M}\right)=0, \quad M=\overline{1, \infty}
$$

which can be rewritten as

$$
\frac{d}{d t}\left(\frac{k-1}{k} \int_{I^{M}}\left(\omega^{M}\right)^{k / 2} d \mathbf{x}-\varepsilon^{M} \frac{k}{2} \int_{I^{M}}\left(\omega^{M}\right)^{k / 2-1}\right)+\sum_{\alpha=1}^{n}\left(a_{\alpha}\left(\left(u_{x_{\alpha}}^{M}\right)^{2}\right) u_{x_{\alpha}}^{M}, u_{x_{\alpha}}^{M}\right)=0, \quad M=\overline{1, \infty} .
$$

After integration from 0 to $t$ we get

$$
\begin{gathered}
\frac{k-1}{k}\left\|\left(\omega^{M}\right)^{1 / 2}(t)\right\|_{k, I^{M}}^{k}-\varepsilon^{M} \frac{k}{2} \int_{I^{M}}\left(\omega^{M}(t, \mathbf{x})\right)^{k / 2-1} d \mathbf{x}+\sum_{\alpha=1}^{n}\left(a_{\alpha}\left(\left(u_{x_{\alpha}}^{M}\right)^{2}\right) u_{x_{\alpha}}^{M}, u_{x_{\alpha}}^{M}\right) D_{D^{t}}= \\
=\frac{k-1}{k}\left\|\left(\omega^{M}\right)^{1 / 2}(0)\right\|_{k, I^{M}}^{k}-\varepsilon^{M} \frac{k}{2} \int_{I^{M}}\left(\omega^{M}(0, \mathbf{x})\right)^{k / 2-1} d \mathbf{x}, \quad M=\overline{1, \infty} .
\end{gathered}
$$

We choose the numbers $\varepsilon^{M} \leqslant 1 / M$ to satisfy inequalities

$$
\text { mes } I^{M} \leqslant\left(\varepsilon^{M}\right)^{-k / 4}, \quad M=\overline{1, \infty} .
$$

Employing (38), (33), we deduce the inequalities

$$
\begin{gathered}
\left\|\left(\omega^{M}\right)^{1 / 2}(0)\right\|_{k, I^{M}} \leqslant\left\|\left|u^{M}(0)\right|+\left(\frac{k}{2} \varepsilon^{M}\right)^{1 / 2}\right\|_{k, I^{M}} \leqslant\left\|u^{M}(0)\right\|_{k}+ \\
+\left(\frac{k}{2} \varepsilon^{M}\right)^{1 / 2}\left(\operatorname{mes} I^{M}\right)^{1 / k} \leqslant E_{1}+\left(\frac{k}{2}\right)^{1 / 2}\left(\varepsilon^{M}\right)^{1 / 4} \leqslant E_{1}+\left(\frac{k}{2}\right)^{1 / 2}, \\
\frac{k}{2} \varepsilon^{M} \int_{I^{M}}\left(\omega^{M}(t, \mathbf{x})\right)^{k / 2-1} d \mathbf{x} \leqslant\left(\frac{k}{2} \varepsilon^{M}\right)^{k / 2} \operatorname{mes} I^{M} \leqslant\left(\frac{k}{2}\right)^{k / 2}\left(\varepsilon^{M}\right)^{k / 4} \leqslant\left(\frac{k}{2}\right)^{k / 2} .
\end{gathered}
$$

Taking into consideration (4) and mimicking (39), (40), (37), for $t \geq 0$ we obtain

$$
\left\|\left(\omega^{M}\right)^{1 / 2}(t)\right\|_{k}^{k}+\sum_{\alpha=1}^{n}\left\|u_{x_{\alpha}}^{M}\right\|_{p_{\alpha}, D^{t}}^{p_{\alpha}} \leqslant E_{2}, \quad M=\overline{1, \infty} .
$$

Moreover, inequalities (4), (41) allows one to establish the estimates

$$
\sum_{\alpha=1}^{n}\left\|a_{\alpha}\left(\left(u_{x_{\alpha}}^{M}\right)^{2}\right) u_{x_{\alpha}}^{M}\right\|_{p_{\alpha} /\left(p_{\alpha}-1\right), D^{t}} \leq \widehat{a} \sum_{\alpha=1}^{n}\left\|u_{x_{\alpha}}^{M}\right\|_{p_{\alpha}, D^{t}}^{p_{\alpha}-1} \leqslant E_{3}, \quad M=\overline{1, \infty} .
$$

Hereinafter the constants $E_{i}$ depend only on $\widehat{a}, \bar{a}, \widehat{b}, \mathbf{p},\|\varphi\|_{W_{k, \mathbf{p}}{ }^{1}(\Omega)}$.

Let us show that all possible solutions to problem (31), (35) are uniformly bounded as $t \geq 0$. Indeed, employing (41), for $t \geq 0$ we deduce

$$
\begin{gathered}
\sum_{j=1}^{M}\left|c_{j}^{M}(t)\right|^{2}=\left\|u^{M}(t)\right\|^{2}= \\
=\int_{\Omega}\left|u^{M}(t)\right|^{k}\left|u^{M}(t)\right|^{2-k} d \mathbf{x} \leqslant\left\|u^{M}(t)\right\|_{k}^{k} \max _{\mathbf{x} \in I^{M}}\left|\sum_{j=1}^{M} c_{j}^{M}(t) \psi_{j}(\mathbf{x})\right|^{2-k} \leqslant
\end{gathered}
$$




$$
\leqslant E_{2}\left(\sum_{j=1}^{M}\left|c_{j}^{M}(t)\right|^{2}\right)^{(2-k) / 2}\left(\sum_{j=1}^{M} m_{j}^{2}\right)^{(2-k) / 2}
$$

It yields

$$
\left|c_{i}^{M}(t)\right|^{k} \leq\left(\sum_{j=1}^{M}\left|c_{j}^{M}(t)\right|^{2}\right)^{k / 2} \leqslant E_{2}\left(\sum_{j=1}^{M} m_{j}^{2}\right)^{(2-k) / 2} \quad, \quad i=\overline{1, M} .
$$

In view of the continuity of the right hand side of equations (35), there exist absolute continuous functions $c_{i}^{M}(t), t \in[0, \infty), i=\overline{1, M}$, which almost everywhere satisfy system (35) and initial condition (31) (see [11, Ch. VIII, Sec. 8]).

We multiply $j$ th equation in 30 by $\frac{d}{d t} c_{j}^{M}(t)$ and sum up then all the equations in $j$ from 1 to $M$ that results in the identities

$$
\left(\left(\left(\omega^{M}\right)^{k / 2-1} u^{M}\right)_{t}, u_{t}^{M}\right)+\sum_{\alpha=1}^{n}\left(a_{\alpha}\left(\left(u_{x_{\alpha}}^{M}\right)^{2}\right) u_{x_{\alpha}}^{M}, u_{t x_{\alpha}}^{M}\right)=0, \quad M=\overline{1, \infty},
$$

which can be rewritten as

$$
\begin{gathered}
(k-1)\left\|\left(\omega^{M}\right)^{k / 4-1} u_{t}^{M} u^{M}\right\|^{2}+\varepsilon^{M} \frac{k}{2}\left\|\left(\omega^{M}\right)^{k / 4-1} u_{t}^{M}\right\|^{2}+ \\
+\frac{1}{2} \frac{d}{d t} \sum_{\alpha=1}^{n} \int_{\Omega} A_{\alpha}\left(\left(u_{x_{\alpha}}^{M}(t)\right)^{2}\right) d \mathbf{x}=0, \quad M=\overline{1, \infty} .
\end{gathered}
$$

After integration from 0 to $t$, employing (21), we get

$$
\begin{gathered}
(k-1)\left\|\left(\omega^{M}\right)^{k / 4-1} u_{t}^{M} u^{M}\right\|_{D^{t}}^{2}+\varepsilon^{M} \frac{k}{2}\left\|\left(\omega^{M}\right)^{k / 4-1} u_{t}^{M}\right\|_{D^{t}}^{2}+ \\
+\frac{1}{2 \widehat{b}} \sum_{\alpha=1}^{n}\left(a_{\alpha}\left(\left(u_{x_{\alpha}}^{M}(t)\right)^{2}\right) u_{x_{\alpha}}^{M}(t), u_{x_{\alpha}}^{M}(t)\right) \leqslant \\
\leqslant \frac{1}{p_{1}} \sum_{\alpha=1}^{n}\left(a_{\alpha}\left(\left(u_{x_{\alpha}}^{M}(0)\right)^{2}\right) u_{x_{\alpha}}^{M}(0), u_{x_{\alpha}}^{M}(0)\right), \quad M=\overline{1, \infty} .
\end{gathered}
$$

Further, due to the inequalities $(k-1)\left(u^{M}\right)^{2}+\varepsilon^{M} \frac{k}{2} \geq(k-1) \omega^{M}$, applying (4) and using (33), we deduce

$$
\left\|\left(\omega^{M}\right)^{(k-2) / 4} u_{t}^{M}\right\|_{D^{t}}^{2}+\sum_{\alpha=1}^{n}\left\|u_{x_{\alpha}}^{M}(t)\right\|_{p_{\alpha}}^{p_{\alpha}} \leqslant E_{5}, \quad M=\overline{1, \infty} .
$$

Let $T$ be an arbitrary positive number. Inequalities (41), 44) imply the boundedness of the sequence $\left\{\left(\omega^{M}\right)^{1 / 2}\right\}_{M=1}^{\infty}$ in the spaces $C\left([0, \infty), L_{k}(\Omega)\right), \quad L_{k}\left(D^{T}\right)$ and the same for the sequence $\left\{u^{M}\right\}_{M=1}^{\infty}$ in the space $C([0, \infty), \stackrel{\circ}{W} \underset{k, \mathbf{p}}{1}(\Omega)), \stackrel{\circ}{W}_{k, \mathbf{p}}^{0,1}\left(D^{T}\right)$ and for the sequence $\left\{\left(\omega^{M}\right)^{(k-2) / 4} u_{t}^{M}\right\}_{M=1}^{\infty}$ in $L_{2}\left(D^{T}\right)$. Moreover, inequalities 42 yield the boundedness of the sequences $a_{\alpha}\left(\left(u_{x_{\alpha}}^{M}\right)^{2}\right) u_{x_{\alpha}}^{M}$ in the spaces $L_{p_{\alpha} /\left(p_{\alpha}-1\right)}\left(D^{T}\right), \alpha=\overline{1, n}$. These facts ensure the selective weak convergence of the mentioned sequences as $M \rightarrow \infty$ in the following spaces,

$$
\begin{gathered}
u^{M} \rightarrow u \quad \text { in } \quad \stackrel{\circ}{W_{k, \mathbf{p}}^{0,1}\left(D^{T}\right),} \\
a_{\alpha}\left(\left(u_{x_{\alpha}}^{M}\right)^{2}\right) u_{x_{\alpha}}^{M} \rightarrow b_{\alpha} \quad \text { in } \quad L_{p_{\alpha} /\left(p_{\alpha}-1\right)}\left(D^{T}\right), \quad \alpha=\overline{1, n} .
\end{gathered}
$$


Moreover, consider the sequence $v^{M}=\left(\omega^{M}\right)^{(k-2) / 4} u^{M}, M=\overline{1, \infty}$, and the sequence of its derivatives $v_{t}^{M}=\left(\omega^{M}\right)^{(k-6) / 4} u_{t}^{M}\left(\frac{k-2}{2} u^{2}+\omega^{M}\right), \quad M=\overline{1, \infty}$. It is obvious that (44) implies inequalities

$$
\left\|v_{t}^{M}\right\|_{D^{t}} \leqslant \frac{k}{2}\left\|\left(\omega^{M}\right)^{(k-2) / 4} u_{t}^{M}\right\|_{D^{t}} \leqslant E_{6}, \quad M=\overline{1, \infty}
$$

which yield the selective weak convergence

$$
v_{t}^{M} \rightarrow g \quad \text { in } \quad L_{2}\left(D^{T}\right) .
$$

In what follows we shall prove that $u^{M}$ selectively a.e. in $D$ converges to $u$ and it will allow us to establish that $g=\left(|u|^{(k-2) / 2} u\right)_{t}$.

The sequence $u^{M} \in C\left([0, \infty), \stackrel{\circ}{W} \frac{1}{k, \mathbf{p}}(\Omega)\right), M=\overline{1, \infty}$, is bounded in this space. For each bounded domain $Q \subset \Omega$ with a smooth boundary we have a compact embedding $L_{1}(Q) \subset$ $W_{1}^{1}(Q)$. This is why by a diagonal process one can establish a strong selective convergence $u^{M}\left(t_{j}, \mathbf{x}\right) \rightarrow h\left(t_{j}, \mathbf{x}\right)$ in $L_{1}(Q)$ on a countable dense set $\left\{t_{j}\right\}_{j=1}^{\infty} \subset[0, T]$. We shall assume that $0, T \in\left\{t_{j}\right\}_{j=1}^{\infty}$. One can also assume that $u^{M}\left(t_{j}, \mathbf{x}\right) \rightarrow h\left(t_{j}, \mathbf{x}\right)$ selectively a.e. in $Q$ for each $t_{j}, j=\overline{1, \infty}$. Completely in the same way as $k \leq p_{1}$ we can assume that the sequence $u^{M}\left(t_{j}, \mathbf{x}\right) \rightarrow h\left(t_{j}, \mathbf{x}\right)$ strongly in $L_{k}(Q)$ for each $t_{j}, j=\overline{1, \infty}$.

Following J.L. Lions [10, Ch. I, Sec. 12.2], let us prove the selective strong convergence of the sequence $v^{M}=\left(\omega^{M}\right)^{(k-2) / 4} u^{M}$ in the space $C\left([0, T], L_{1}(Q)\right)$. First, applying (45), let us prove the equicontinuity in $t$ of the sequence $v^{M}$ in $L_{2}(\Omega)$,

$$
\begin{gathered}
\left\|v^{M}\left(t_{2}\right)-v^{M}\left(t_{1}\right)\right\|=\left\|\int_{t_{1}}^{t_{2}} v_{t}^{M}(t) d t\right\| \leqslant \int_{t_{1}}^{t_{2}}\left\|v_{t}^{M}(t)\right\| d t \leqslant \\
\leqslant\left|t_{2}-t_{1}\right|^{1 / 2}\left(\int_{t_{1}}^{t_{2}}\left\|v_{t}^{M}(t)\right\|^{2} d t\right)^{1 / 2} \leqslant E_{6}\left|t_{2}-t_{1}\right|^{1 / 2}, \quad t_{1}, t_{2} \in[0, T], \quad M=\overline{1, \infty} .
\end{gathered}
$$

Inequalities (41) imply the uniform in $t \in[0, T]$ boundedness of the sequence $v^{M}(t, \mathbf{x})$ in $L_{2}(\Omega)$,

$$
\left\|v^{M}(t)\right\|=\left\|\left(\omega^{M}\right)^{(k-2) / 4} u^{M}(t)\right\| \leqslant\left\|\left(\omega^{M}\right)^{k / 4}\right\|=\left\|\left(\omega^{M}\right)^{1 / 2}\right\|_{k}^{k / 2} \leqslant E_{7}, \quad M=\overline{1, \infty} .
$$

Due to the boundedness of the sequence $v^{M}(t, \mathbf{x}), M=\overline{1, \infty}$, in the space $C\left([0, T], L_{2}(\Omega)\right)$, it selectively weakly converges in $L_{2}(\Omega)$ for the same $t_{j}$ as above. The established selective convergence $u^{M}\left(t_{j}, \mathbf{x}\right) \rightarrow h\left(t_{j}, \mathbf{x}\right)$ a.e. in $Q$ for each $t_{j}$ implies selective convergence $v^{M}\left(t_{j}, \mathbf{x}\right) \rightarrow$ $v\left(t_{j}, \mathbf{x}\right)=\left|h\left(t_{j}, \mathbf{x}\right)\right|^{(k-2) / 2} h\left(t_{j}, \mathbf{x}\right)$ a.e. in $Q$. Then, by Egorov theorem, for each $\delta>0$ we obtain the uniform convergence $v^{M}\left(t_{j}, \mathbf{x}\right) \rightrightarrows v\left(t_{j}, \mathbf{x}\right)$ on $Q_{\delta}$, mes $\left(Q \backslash Q_{\delta}\right)<\delta$. By inequalities

$$
\begin{gathered}
\left\|v^{M}\left(t_{j}\right)-v\left(t_{j}\right)\right\|_{1, Q} \leqslant \operatorname{mes} Q \max _{\mathbf{x} \in Q_{\delta}}\left|v^{M}\left(t_{j}, \mathbf{x}\right)-v\left(t_{j}, \mathbf{x}\right)\right|+\left\|v^{M}\left(t_{j}\right)-v\left(t_{j}\right)\right\|_{1, Q \backslash Q_{\delta}} \leqslant \\
\leqslant \operatorname{mes} Q \max _{\mathbf{x} \in Q_{\delta}}\left|v^{M}\left(t_{j}, \mathbf{x}\right)-v\left(t_{j}, \mathbf{x}\right)\right|+\delta^{1 / 2}\left\|v^{M}\left(t_{j}\right)-v\left(t_{j}\right)\right\|_{2, Q \backslash Q_{\delta}}
\end{gathered}
$$

it implies the strong convergence $v^{M}\left(t_{j}, \mathbf{x}\right) \rightarrow v\left(t_{j}, \mathbf{x}\right)$ in $L_{1}(Q)$ for each $t_{j}$.

For a bounded domain $Q$ by 46 one can easily establish the uniform fundamentality of the sequence $v^{M}(t, \mathbf{x})$ in the norm of $L_{1}(Q)$,

$$
\begin{gathered}
\left\|v^{N}(t)-v^{M}(t)\right\|_{1, Q}=\left\|v^{N}(t)-v^{N}\left(t_{j_{l}}\right)+v^{N}\left(t_{j_{l}}\right)-v^{M}\left(t_{j_{l}}\right)+v^{M}\left(t_{j_{l}}\right)-v^{M}(t)\right\|_{1, Q} \leqslant \\
\leqslant 2(\operatorname{mes} Q)^{1 / 2} E_{6}\left|t-t_{j_{l}}\right|^{1 / 2}+\left\|v^{N}\left(t_{j_{l}}\right)-v^{M}\left(t_{j_{l}}\right)\right\|_{1, Q} .
\end{gathered}
$$

Choosing a finite set of the numbers $t_{j_{l}}$ with a small step and increasing then $N, M$, we achieve the uniform in $t$ smallness of the right hand side. 
Thus, we have established the selective strong convergence of $v^{M} \rightarrow v$ in $C\left([0, T], L_{1}(Q)\right)$. The convergence holds also in $L_{1}((0, T) \times Q)$, and hence $v^{M} \rightarrow v$ selectively converges a.e. in $(0, T) \times Q$. Due to the arbitrariness of $Q$, the sequence $v^{M}$ selectively converges to $v$ a.e. in $D^{T}$. Moreover, due to the the arbitrariness of $T$, choosing $T=1,2, \ldots$, by a diagonal process one can select a subsequence $v^{M} \rightarrow v$ a.e. in $D$ as $M \rightarrow \infty$. Then the subsequence $u^{M}(t, \mathbf{x})$ selectively converges to $h(t, \mathbf{x})$ a.e. in $D$. According to Lemma $2, u^{M}(t, \mathbf{x}) \rightarrow h(t, \mathbf{x})$ in $L_{k}\left(D^{T}\right)$ for any $T>0$, and by the uniqueness of the limit $h(t, \mathbf{x})=u(t, \mathbf{x})$ a.e. in $D$. Thus, $v^{M}$ selectively converges to $v=|u|^{(k-2) / 2} u$ a.e. in $D$.

According to Lemma 2, $v^{M} \rightarrow v$ weakly in $L_{2}\left(D^{T}\right)$. Further, $\left(v_{t}^{M}, w\right)_{D^{T}}=-\left(v^{M}, w_{t}\right)_{D^{T}}$ for each function $w \in C_{0}^{\infty}\left(D^{T}\right)$, and passing to the limit as $M \rightarrow \infty$, we obtain

$$
(g, w)_{D^{T}}=-\left(v, w_{t}\right)_{D^{T}} .
$$

It follows that $g=v_{t}=\left(|u|^{(k-2) / 2} u\right)_{t}$. We observe that the belonging $v, v_{t} \in L_{2}\left(D^{T}\right)$ implies $v \in C\left([0, \infty), L_{2}(\Omega)\right)$.

Let us show that the sequence $u_{t}^{M}, M=\overline{1, \infty}$, is bounded in $L_{k}\left(D^{T}\right)$. Indeed, it follows from (41), (44) that

$$
\begin{gathered}
\left\|u_{t}^{M}\right\|_{k, D^{T}}=\left(\int_{D^{T}}\left(\omega^{M}\right)^{k(k-2) / 4}\left|u_{t}^{M}\right|^{k}\left(\omega^{M}\right)^{(2-k) k / 4} d \mathbf{x} d t\right)^{1 / k} \leqslant \\
\leqslant\left\|\left(\omega^{M}\right)^{(k-2) / 4} u_{t}^{M}\right\|_{2, D^{T}}\left\|\left(\omega^{M}\right)^{1 / 2}\right\|_{k, D^{T}}^{(2-k) / 2} \leqslant E_{8} .
\end{gathered}
$$

The boundedness of $\left\|u_{t}^{M}\right\|_{k, D^{T}}$ implies $u_{t}^{M} \rightarrow b$ in $L_{k}\left(D^{T}\right)$. Then $\left(u_{t}^{M}, w\right)_{D^{T}}=-\left(u^{M}, w_{t}\right)_{D^{T}}$ for each function $w \in C_{0}^{\infty}\left(D^{T}\right)$, and passing to the limit as $M \rightarrow \infty$, we get

$$
(b, w)_{D^{T}}=-\left(u, w_{t}\right)_{D^{T}},
$$

hence, $b=u_{t}$. Then one can assume that $u_{t}^{M} \rightarrow u_{t}$ weakly in $L_{k}\left(D^{T}\right)$. We note that the belonging $u, u_{t} \in L_{k}\left(D^{T}\right)$ yields $u \in C\left([0, \infty), L_{k}(\Omega)\right)$.

On one hand, estimate $(33)$ and the convergence $u^{M}(0, \mathbf{x}) \rightarrow u(0, \mathbf{x})$ a.e. as $M \rightarrow \infty$ by Lemma 2 imply the weak convergence $u^{M}(0, \mathbf{x}) \rightarrow u(0, \mathbf{x})$ in $L_{k}(\Omega)$ as $M \rightarrow \infty$. On the other hand, by the choice $(32), u^{K}(0, \mathbf{x})$ strongly converges to $\varphi(\mathbf{x})$ in $L_{k}(\Omega)$. Due to the uniqueness of the weak limit, $u(0, \mathbf{x})=\varphi(\mathbf{x})$ for a.e. $\mathbf{x} \in \Omega$.

Let us prove that the function $u(t, \mathbf{x})$ satisfies integral identity. (18). Equations (30) imply the identities

$$
\left(\left(\left(\omega^{M}\right)^{(k-2) / 2} u^{M}\right)_{t}, w\right)_{D^{T}}+\sum_{\alpha=1}^{n}\left(a_{\alpha}\left(\left(u_{x_{\alpha}}^{M}\right)^{2}\right) u_{x_{\alpha}}^{M}, w_{x_{\alpha}}\right)_{D^{T}}=0, \quad M=\overline{1, \infty},
$$

satisfied for each funcion $w(\tau, \mathbf{x}) \in P=\bigcup_{L=1}^{\infty} P_{L}$. We integrate the first term by parts,

$$
\begin{aligned}
& \left.\left(\left(\omega^{M}\right)^{(k-2) / 2} u^{M}, w\right)\right|_{t=0} ^{t=T}-\left(\left(\omega^{M}\right)^{(k-2) / 2} u^{M}, w_{t}\right)_{D^{T}}+ \\
& +\sum_{\alpha=1}^{n}\left(a_{\alpha}\left(\left(u_{x_{\alpha}}^{M}\right)^{2}\right) u_{x_{\alpha}}^{M}, w_{x_{\alpha}}\right)_{D^{T}}=0, \quad M=\overline{1, \infty} .
\end{aligned}
$$

We observe that the inequality $\left(\omega^{M}\right)^{(k-2) / 2}\left|u^{M}\right| \leqslant\left(\omega^{M}\right)^{(k-1) / 2} \in C\left([0, \infty), L_{k^{\prime}}(\Omega)\right)$ holds true, since $\left\|\left(\omega^{M}\right)^{(k-1) / 2}\right\|_{k^{\prime}}=\left\|\left(\omega^{M}\right)^{1 / 2}\right\|_{k}^{k-1}$ is a bounded sequence in $C[0, \infty)$. Hence, by Lemma 2 , the convergence $\left(\omega^{M}\right)^{(k-2) / 2} u^{M}$ selectively weakly converges to $|u|^{k-2} u$ in $L_{k^{\prime}}\left(D^{T}\right)$ and $\left(\omega^{M}(T)\right)^{(k-2) / 2} u^{M}(T) \rightarrow|u(T)|^{k-2} u(T), \quad\left(\omega^{M}(0)\right)^{(k-2) / 2} u^{M}(0) \rightarrow|u(0)|^{k-2} u(0)$ in $L_{k^{\prime}}(\Omega)$. One can also state that $\left(\omega^{M}(T)\right)^{1 / 2} \rightarrow|u(T)|,\left(\omega^{M}(0)\right)^{1 / 2} \rightarrow|u(0)|$ in $L_{k}(\Omega)$. The fact that the 
limiting functions look exactly like this is justified by the above proven convergence of the subsequence $u^{M}$ a.e. in $D^{T}$, and also a.e. in $\Omega$ for $t=0, T$.

In (48) we can pass to the limit as $M \rightarrow \infty$ that results in the identity

$$
\left.\left(|u|^{k-2} u, w\right)\right|_{t=0} ^{t=T}-\left(|u|^{k-2} u, w_{t}\right)_{D^{T}}+\sum_{\alpha=1}^{n}\left(b_{\alpha}, w_{x_{\alpha}}\right)_{D^{T}}=0
$$

valid for each function $w \in P$. Since $P$ is dense in the space $\stackrel{\circ}{W}_{k, \mathbf{p}}^{1,1}\left(D^{T}\right)$ (Lemma 3), identity 49 holds true for arbitrary $w \in \stackrel{\circ}{W_{k, \mathbf{p}}^{1,1}}\left(D^{T}\right)$. Here we employ that $|u|^{k-2} u \in L_{k^{\prime}}\left(D^{T}\right), \quad b_{\alpha} \in$ $L_{p_{\alpha} /\left(p_{\alpha}-1\right)}\left(D^{T}\right), \alpha=\overline{1, n}$. In particular, for $w=u$, we employ the identity

$$
\int_{0}^{t}\left(|u|^{k-2} u, u_{\tau}\right) d \tau=\left.\frac{1}{k}\|u(t)\|_{k}^{k}\right|_{t=0} ^{t=T}
$$

to get

$$
\begin{gathered}
\sum_{\alpha=1}^{n}\left(b_{\alpha}, u_{x_{\alpha}}\right)_{D^{T}}+\left.\|u(t)\|_{k}^{k}\right|_{t=0} ^{t=T}-\left(|u|^{k-2} u, u_{t}\right)_{D^{T}}= \\
=\left.\frac{k-1}{k}\|u(t)\|_{k}^{k}\right|_{t=0} ^{t=T}+\sum_{\alpha=1}^{n}\left(b_{\alpha}, u_{x_{\alpha}}\right)_{D^{T}}=0 .
\end{gathered}
$$

Let us show that for each function $v \in \stackrel{\circ}{W_{k, \mathbf{p}}^{1,1}}\left(D^{T}\right)$ the identity

$$
\sum_{\alpha=1}^{n}\left(b_{\alpha}, v_{x_{\alpha}}\right)_{D^{T}}=\sum_{\alpha=1}^{n}\left(a_{\alpha}\left(\left(u_{x_{\alpha}}\right)^{2}\right) u_{x_{\alpha}}, v_{x_{\alpha}}\right)_{D^{T}}
$$

holds true. We deduct identity (48) from 37 with $t=T$ and for $w \in P$ we obtain the relations

$$
\begin{gathered}
-\left.\left(\left(\omega^{M}\right)^{(k-2) / 2} u^{M}, w\right)\right|_{t=0} ^{t=T}+\left(\left(\omega^{M}\right)^{(k-2) / 2} u^{M}, w_{t}\right)_{D^{T}}+ \\
+\sum_{\alpha=1}^{n}\left(a_{\alpha}\left(\left(u_{x_{\alpha}}^{M}\right)^{2}\right) u_{x_{\alpha}}^{M},\left(u^{M}-w\right)_{x_{\alpha}}\right)_{D^{T}}+ \\
+\left.\frac{k-1}{k}\left\|\left(\omega^{M}\right)^{1 / 2}(t)\right\|_{k}^{k}\right|_{t=0} ^{t=T}-\left.\varepsilon^{M} \frac{k}{2} \int_{I^{M}}\left(\omega^{M}(t, \mathbf{x})\right)^{k / 2-1} d \mathbf{x}\right|_{t=0} ^{t=T}=0, \quad M=\overline{1, \infty},
\end{gathered}
$$

that by the condition of monotonic non-decay of the functions $a_{\alpha}\left(z^{2}\right) z, z \in \mathbb{R}, \alpha=\overline{1, n}$, (see (20)) and inequality (40) imply the estimates

$$
\begin{gathered}
-\left.\left(\left(\omega^{M}\right)^{(k-2) / 2} u^{M}, w\right)\right|_{t=0} ^{t=T}+\left(\left(\omega^{M}\right)^{(k-2) / 2} u^{M}, w_{t}\right)_{D^{T}}+ \\
+\sum_{\alpha=1}^{n}\left(a_{\alpha}\left(\left(w_{x_{\alpha}}\right)^{2}\right) w_{x_{\alpha}},\left(u^{M}-w\right)_{x_{\alpha}}\right)_{D^{T}}+ \\
+\left.\frac{k-1}{k}\left\|\left(\omega^{M}\right)^{1 / 2}(t)\right\|_{k}^{k}\right|_{t=0} ^{t=T}-\left(\varepsilon^{M}\right)^{k / 4}\left(\frac{k}{2}\right)^{k / 2} \leqslant 0, \quad M=\overline{1, \infty} .
\end{gathered}
$$

Further, we pass to the limit as $M \rightarrow \infty$ for a fixed $w \in P$ employing at that the above proven convergence. 
Thus, for arbitrary $w \in P$ the inequality

$$
\begin{gathered}
-\left.\left(|u|^{k-2} u, w\right)\right|_{t=0} ^{t=T}+\left(|u|^{k-2} u, w_{t}\right)_{D^{T}}+\sum_{\alpha=1}^{n}\left(a_{\alpha}\left(w_{x_{\alpha}}^{2}\right) w_{x_{\alpha}},(u-w)_{x_{\alpha}}\right)_{D^{T}}+ \\
+\left.\frac{k-1}{k}\|u(t)\|_{k}^{k}\right|_{t=0} ^{t=T} \leqslant 0
\end{gathered}
$$

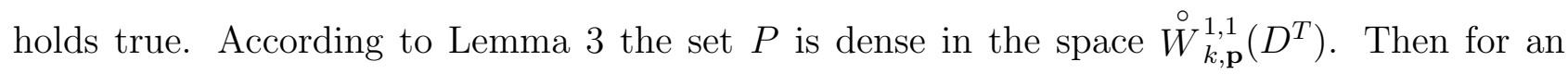
arbitrary function $w \in \stackrel{\circ}{W_{k, \mathbf{p}}^{1,1}}\left(D^{T}\right)$ there exists a sequence $w^{l} \in P$ such that $\left\|w^{l}-w\right\|_{W_{k, \mathbf{p}}^{1,1}\left(D^{T}\right)} \rightarrow 0$ as $l \rightarrow \infty$. We write $(53)$ for $w=w^{l}$ and pass then to the limit as $l \rightarrow \infty$. Justification of passage to the limits as $l \rightarrow \infty$

$$
\left(a_{\alpha}\left(\left(w_{x_{\alpha}}^{l}\right)^{2}\right) w_{x_{\alpha}}^{l},\left(u-w^{l}\right)_{x_{\alpha}}\right)_{D^{T}} \rightarrow\left(a_{\alpha}\left(w_{x_{\alpha}}^{2}\right) w_{x_{\alpha}},(u-w)_{x_{\alpha}}\right)_{D^{T}}, \quad \alpha=\overline{1, n},
$$

was provided in [6]. Thus, identity 53 is established for an arbitrary $w \in \stackrel{\circ}{W}_{k, \mathbf{p}}^{1,1}\left(D^{T}\right)$.

We deduct (51) from (53) and add (49) that yields the inequality

$$
\sum_{\alpha=1}^{n}\left(a_{\alpha}\left(w_{x_{\alpha}}^{2}\right) w_{x_{\alpha}}-b_{\alpha},(u-w)_{x_{\alpha}}\right)_{D^{T}} \leq 0,
$$

being true for $w \in \stackrel{\circ}{W_{k, \mathbf{p}}^{1,1}}\left(D^{T}\right)$. In 54 we let $w=u+\varepsilon v, \varepsilon>0$, where $v \in \stackrel{\circ}{W_{k, \mathbf{p}}^{1,1}}\left(D^{T}\right)$ that gives us

$$
\sum_{\alpha=1}^{n}\left(a_{\alpha}\left(\left(u_{x_{\alpha}}+\varepsilon v_{x_{\alpha}}\right)^{2}\right)\left(u_{x_{\alpha}}+\varepsilon v_{x_{\alpha}}\right)-b_{\alpha}, v_{x_{\alpha}}\right)_{D^{T}} \geq 0 .
$$

The latter inequality as $\varepsilon \rightarrow 0$ yields the relation

$$
\sum_{\alpha=1}^{n}\left(a_{\alpha}\left(u_{x_{\alpha}}^{2}\right) u_{x_{\alpha}}-b_{\alpha}, v_{x_{\alpha}}\right)_{D^{T}} \geq 0
$$

which by the arbitrariness of $v$ lead us to identity 52 . By 49 and 52 for $v \in \stackrel{\circ}{W}_{k, \mathbf{p}}^{1,1}\left(D^{T}\right)$ we conclude that

$$
-\left(|u|^{k-2} u, v_{t}\right)_{D^{T}}+\sum_{\alpha=1}^{n}\left(a_{\alpha}\left(u_{x_{\alpha}}^{2}\right) u_{x_{\alpha}}, v_{x_{\alpha}}\right)_{D^{T}}+\left.\left(|u|^{k-2} u, v\right)\right|_{t=0} ^{t=T}=0 .
$$

Thus, (18) is proven.

By (51), (52) we get the identity

$$
\frac{k-1}{k}\|u(t)\|_{k}^{k}+\sum_{\alpha=1}^{n} \int_{0}^{t}\left(a_{\alpha}\left(u_{x_{\alpha}}^{2}\right) u_{x_{\alpha}}, u_{x_{\alpha}}\right) d \tau=\frac{k-1}{k}\|\varphi\|_{k}^{k}, \quad t \geq 0,
$$

differentiating which w.r.t. $t$, we obtain

$$
\frac{k-1}{k} \frac{d}{d t}\|u(t)\|_{k}^{k}+\sum_{\alpha=1}^{n}\left(a_{\alpha}\left(u_{x_{\alpha}}^{2}\right) u_{x_{\alpha}}, u_{x_{\alpha}}\right)=0, \quad t>0 .
$$

Applying then (4), by (56), (57) we get (28), (29).

Proposition 1. A generalized solution $u(t, \mathbf{x})$ of problem (1) -(3) with a bounded initial function $\varphi(\mathbf{x}) \in L_{\infty}(\Omega) \cap \stackrel{\circ}{W} \underset{k, \mathbf{p}}{1}(\Omega)$ is bounded, i.e.,

$$
\text { vrai } \sup _{D}|u(t, \mathbf{x})| \leqslant B<\infty .
$$

We omit the proof of this proposition. 


\section{Admissible DECAY RATE OF SOLUTION}

Since the unique solvability of the problem (1)-(3) is not established, we in fact obtain the lower estimate for the constructed solution.

Proof of Theorem 2. We first assume that the domain $\Omega$ is bounded and let us prove estimate (8) for Galerkin approximations.

We introduce the notations

$$
\begin{gathered}
G^{M}(t)=\sum_{\alpha=1}^{n} \int_{\Omega} a_{\alpha}\left(\left(u_{x_{\alpha}}^{M}\right)^{2}\right)\left(u_{x_{\alpha}}^{M}\right)^{2} d \mathbf{x}, \quad H^{M}(t)=\sum_{\alpha=1}^{n} \int_{\Omega} A_{\alpha}\left(\left(u_{x_{\alpha}}^{M}\right)^{2}\right) d \mathbf{x}, \\
E^{M}(t)=\int_{I^{M}}\left(\frac{k-1}{k}\left(\omega^{M}(t)\right)^{k / 2}-\varepsilon^{M} \frac{k}{2}\left(\omega^{M}(t)\right)^{(k-2) / 2}\right) d \mathbf{x}+\left(\frac{2}{k}\right)^{1 / 2}\left(\varepsilon^{M}\right)^{k / 4}
\end{gathered}
$$

employing (21), we obtain inequalities

$$
\frac{p_{1}}{2} H^{M}(t) \leqslant G^{M}(t) \leqslant \widehat{b} H^{M}(t), \quad t \geq 0 .
$$

We rewrite identities (36), (43) as

$$
\begin{gathered}
\frac{d E^{M}(t)}{d t}+G^{M}(t)=0, \quad t>0 \\
\int_{I^{M}}\left((k-1)\left(u^{M}\right)^{2}+\frac{k}{2} \varepsilon^{M}\right)\left(\omega^{M}\right)^{(k-4) / 2}\left(u_{t}^{M}\right)^{2}+\frac{1}{2} \frac{d H^{M}(t)}{d t}=0, \quad t>0 .
\end{gathered}
$$

Applying Cauchy-Schwarz inequality for integrals, we obtain the relations

$$
\begin{gathered}
\left(\frac{d E^{M}(t)}{d t}\right)^{2}=\left(\int_{I^{M}}\left((k-1)\left(\omega^{M}\right)^{(k-2) / 2}+\varepsilon^{M} \frac{k}{2}(2-k)\left(\omega^{M}\right)^{(k-4) / 2}\right) u^{M} u_{t}^{M} d \mathbf{x}\right)^{2} \leqslant \\
\leqslant\left((k-1)\left(\int_{I^{M}}\left(\omega^{M}\right)^{(k-4) / 2}\left(u^{M}\right)^{2}\left(u_{t}^{M}\right)^{2}\right)^{1 / 2}\left(\int_{I^{M}}\left(\omega^{M}\right)^{k / 2}\right)^{1 / 2}+\right. \\
\left.+\varepsilon^{M} \frac{k}{2}\left(\int_{I^{M}}\left(\omega^{M}\right)^{(k-4) / 2}\left(u_{t}^{M}\right)^{2}\right)^{1 / 2}\left(\int_{I^{M}}(2-k)\left(\omega^{M}\right)^{(k-2) / 2}\right)^{1 / 2}\right)^{2} .
\end{gathered}
$$

Employing Cauchy-Schwarz inequality for sums, by (61), 40) we deduce

$$
\begin{gathered}
\left(\frac{d E^{M}(t)}{d t}\right)^{2} \leqslant \int_{I^{M}}\left((k-1)\left(u^{M}\right)^{2}+\varepsilon^{M} \frac{k}{2}\right)\left(\omega^{M}\right)^{(k-4) / 2}\left(u_{t}^{M}\right)^{2} d \mathbf{x} \times \\
\times \int_{I^{M}}\left((k-1)\left(\omega^{M}\right)^{k / 2}+(2-k) \varepsilon^{M} \frac{k}{2}\left(\omega^{M}\right)^{(k-2) / 2}\right) d \mathbf{x} \leqslant \\
\leqslant-\frac{1}{2} \frac{d H^{M}(t)}{d t}\left\{\int_{I^{M}}\left((k-1)\left(\omega^{M}\right)^{k / 2}-\varepsilon^{M} \frac{k^{2}}{2}\left(\omega^{M}\right)^{(k-2) / 2}\right) d \mathbf{x}+k\left(\frac{2}{k}\right)^{1 / 2}\left(\varepsilon^{M}\right)^{k / 4}\right\}= \\
=-\frac{k}{2} \frac{d H^{M}(t)}{d t} E(t) .
\end{gathered}
$$


Relations (62), (60), (59) imply inequalities

$$
\frac{k}{2} E^{M}(t) \frac{d H^{M}(t)}{d t} \leqslant \frac{d E^{M}(t)}{d t} G^{M}(t) \leqslant \frac{p_{1}}{2} \frac{d E^{M}(t)}{d t} H^{M}(t),
$$

which we rewrite as

$$
\frac{d H^{M}(t)}{d t} / H^{M}(t) \leqslant \frac{p_{1}}{k} \frac{d E^{M}(t)}{d t} / E^{M}(t) .
$$

Solving differential inequality and applying (59), we obtain the estimates

$$
\frac{1}{\widehat{b}} G^{M}(t) \leqslant H^{M}(t) \leqslant H^{M}(0)\left(E^{M}(t)\right)^{p_{1} / k} /\left(E^{M}(0)\right)^{p_{1} / k}, \quad t>0 .
$$

Mimicking (60), 63), (59), we arrive at the relations

$$
\begin{gathered}
\frac{d E^{M}(t)}{d t} \geq-\widehat{b} H^{M}(0)\left(E^{M}(t)\right)^{p_{1} / k} /\left(E^{M}(0)\right)^{p_{1} / k} \geq \\
\geq-\frac{2 \widehat{b}}{p_{1}} G^{M}(0)\left(E^{M}(t)\right)^{p_{1} / k} /\left(E^{M}(0)\right)^{p_{1} / k},
\end{gathered}
$$

which we rewrite as

$$
\frac{d E^{M}(t)}{d t} /\left(E^{M}(t)\right)^{p_{1} / k} \geq-\frac{2 \widehat{b}}{p_{1}} G^{M}(0) /\left(E^{M}(0)\right)^{p_{1} / k} .
$$

Solving differential inequality, we obtain the estimate

$$
E^{M}(t) \geq E^{M}(0)\left(t \frac{2\left(p_{1}-k\right) \widehat{b}}{k p_{1}} G^{M}(0) / E^{M}(0)+1\right)^{-k /\left(p_{1}-k\right)}, \quad t>0 .
$$

For a fixed $t>0$ as $k \leq p_{1}$ in the case of the bounded domain $\Omega$ the sequence $u^{M}(t, \mathbf{x})$ is selectively strongly converges as $M \rightarrow \infty$ to $u(t, \mathbf{x})$ in the space $L_{k}(\Omega)$. It is obvious that

$$
E^{M}(t) \leqslant \frac{k-1}{k}\left\|\left(\omega^{M}\right)^{1 / 2}(t)\right\|_{k}^{k}+\left(\frac{2}{k}\right)^{1 / 2}\left(\varepsilon^{M}\right)^{k / 4}, \quad M=\overline{1, \infty},
$$

and according to $(38)$, the inequality

$$
\lim _{M \rightarrow \infty} E^{M}(t) \leqslant \frac{k-1}{k}\|u(t)\|_{k}^{k}=E(t)
$$

holds true.

Moreover, due to (40) the inequalities

$$
\begin{gathered}
\lim _{M \rightarrow \infty} E^{M}(0) \geq \lim _{M \rightarrow \infty}\left(\frac{k-1}{k}\left\|u^{M}(0)\right\|_{k}^{k}+\left(\frac{2}{k}\right)^{1 / 2} \frac{2-k}{2}\left(\varepsilon^{M}\right)^{k / 4}\right)=\frac{k-1}{k}\|\varphi\|_{k}^{k}, \\
\lim _{M \rightarrow \infty} G^{M}(0) \leqslant \lim _{M \rightarrow \infty} \widehat{a} \sum_{\alpha=1}^{n}\left\|u_{x_{\alpha}}^{M}\right\|_{p_{\alpha}}^{p_{\alpha}}=\widehat{a} \sum_{\alpha=1}^{n}\left\|\varphi_{x_{\alpha}}\right\|_{p_{\alpha}}^{p_{\alpha}}
\end{gathered}
$$

hold true. After the passage to the limit in (64) as $M \rightarrow \infty$, we obtain

$$
\|u(t)\|_{k}^{k} \geq\|\varphi\|_{k}^{k}\left(1+C\left(\|\varphi\|_{W_{k, \mathbf{p}}^{1}(\Omega)}\right) t\right)^{-k /\left(p_{1}-k\right)} .
$$

Let us establish estimate (65) for a solution to problem (1)-(3) in an unbounded domain $\Omega$. Let $\Omega^{(l)} \subset \Omega$ be bounded subdomains such that $\Omega^{(l)} \subset \Omega^{(l+1)}, l=\overline{1, \infty}, \bigcup_{l=1}^{\infty} \Omega^{(l)}=\Omega$. By $u^{(l)}$ we denote the solutions in $\Omega^{(l)}$ with a compactly supported initial function $\left(\operatorname{supp} \varphi \subset \Omega^{(1)}\right)$; one can assume that these solutions are extended by zero outside $\Omega^{(l)}$. The convergence of the 
sequence $u^{(l)}(t, \mathbf{x})$ to the solution $u(t, \mathbf{x})$ of problem (1)-(3) as $l \rightarrow \infty$ can be shown in the same way as in Theorem 4.

Property (25) ensures the estimate

$$
\left\|u^{(l)}\right\|_{W_{k, \mathbf{p}}^{1}(\Omega)} \leqslant C, \quad t>0, \quad l=\overline{1, \infty} .
$$

Then for a fixed $t>0$ one can assume that $u^{(l)}(t, \mathbf{x}) \rightarrow u(t, \mathbf{x})$ in $\stackrel{\circ}{W}{ }_{k}^{1}\left(\Omega^{r}\right)$ as $l \rightarrow \infty$. Employing the compactness of the embedding $W_{k}^{1}\left(\Omega^{r}\right) \subset L_{k}\left(\Omega^{r}\right)$, we establish the strong convergence $u^{(l)}(t, \mathbf{x}) \rightarrow u(t, \mathbf{x})$ in $L_{k}\left(\Omega^{r}\right)$ as $l \rightarrow \infty$ for each $r>0$. Owing to estimate $(7)$, for any $\varepsilon$ there exists $r$ such that the inequality

$$
\left\|u^{(l)}(t)\right\|_{k, \Omega_{r}}^{k} \leqslant \varepsilon
$$

holds true. The function $u^{(l)}$ satisfies estimate 65 that yields

$$
\left\|u^{(l)}(t)\right\|_{k, \Omega^{r}}^{k} \geq\|\varphi\|_{k}^{k}\left(1+C\left(\|\varphi\|_{W_{k, \mathbf{p}}^{1}(\Omega)}\right) t\right)^{-k /\left(p_{1}-k\right)}-\varepsilon .
$$

Employing the strong convergence in $L_{k}\left(\Omega^{r}\right)$, we pass to the limit as $l \rightarrow \infty$ and then as $r \rightarrow \infty(\varepsilon \rightarrow 0)$. Thus, estimate $(8)$ is proven for the unbounded domain $\Omega$ for arbitrary $t \geq 0$.

\section{UPPER ESTIMATES}

In this section we prove Theorem 1,3.

Proof of Theorem 1. Let $\xi\left(x_{s}\right)$ be a Lipshitz nonnegative cut-off function. In (55) we let $v=u \xi$ and employ (50) to obtain the relation

$$
\left.\frac{k-1}{k} \int_{\Omega}|u|^{k} \xi\right|_{\tau=0} ^{\tau=t} d \mathbf{x}+\sum_{\alpha=1}^{n} \int_{D^{t}} a_{\alpha}\left(u_{x_{\alpha}}^{2}\right) u_{x_{\alpha}}(u \xi)_{x_{\alpha}} d \mathbf{x} d \tau=0 .
$$

Using (4), we obtain (taking into consideration that $\xi \varphi=0$ )

$$
\begin{gathered}
\frac{k-1}{k} \int_{\Omega}|u(t, \mathbf{x})|^{k} \xi\left(x_{s}\right) d \mathbf{x}+\bar{a} \sum_{\alpha=1}^{n} \int_{D^{t}} \xi\left|u_{x_{\alpha}}\right|^{p_{\alpha}} d \mathbf{x} d \tau \leqslant \\
\leqslant \widehat{a} \int_{D^{t}}|u|\left|u_{x_{s}}\right|^{p_{s}-1} \xi^{\prime}\left(x_{s}\right) d \mathbf{x} d \tau \equiv I^{t} .
\end{gathered}
$$

Let $\theta(x), x>0$, be an absolute continuous function being one as $x \geq 1$, vanishing as $x \leq 0$, and being linear as $x \in[0,1]$. In 66 we let $\xi\left(x_{s}\right)=\theta\left(\left(x_{s}-r\right) / \rho\right)$. It is clear that

$$
\xi^{\prime}\left(x_{s}\right)=\frac{1}{\rho}, \quad x \in(r, r+\rho), \quad \xi^{\prime}\left(x_{s}\right)=0, \quad x \notin(r, r+\rho) .
$$

Let us estimate the integral

$$
I^{t}=\frac{\widehat{a}}{\rho} \int_{0}^{t} \int_{\Omega_{r}^{r+\rho}}|u|\left|u_{x_{s}}\right|^{p_{s}-1} d \mathbf{x} d \tau .
$$

Employing Young inequality and (58), for each $\varepsilon>0$ we deduce

$$
I^{t} \leqslant \frac{\widehat{a}}{\varepsilon \rho}\left(\frac{p_{s}-1}{p_{s}} \int_{0}^{t} \int_{\Omega_{r}^{r+\rho}}\left|u_{x_{s}}\right|^{p_{s}} d \mathbf{x} d \tau+\frac{\varepsilon^{p_{s}}}{p_{s}} B^{p_{s}-k} \int_{0}^{t} \int_{\Omega_{r}^{r+\rho}}|u|^{k} d \mathbf{x} d \tau\right) .
$$


Mimicking (66), (68), we obtain the inequality

$$
\begin{aligned}
& \frac{k-1}{k} \int_{\Omega_{r+\rho}}|u(t, \mathbf{x})|^{k} d \mathbf{x}+\bar{a} \sum_{\alpha=1}^{n} \int_{0}^{t} \int_{\Omega_{r+\rho}}\left|u_{x_{\alpha}}\right|^{p_{\alpha}} d \mathbf{x} d \tau \leqslant \\
& \leqslant \frac{C_{1}}{\varepsilon \rho}\left(\int_{0}^{t} \int_{\Omega_{r}^{r+\rho}}\left|u_{x_{s}}\right|^{p_{s}} d \mathbf{x} d \tau+\varepsilon^{p_{s}} \int_{0}^{t} \int_{\Omega_{r}^{r+\rho}}|u|^{k} d \mathbf{x} d \tau\right) .
\end{aligned}
$$

We introduce the notation

$$
F_{r}(t)=\int_{\Omega_{r}}|u(t, \mathbf{x})|^{k} d \mathbf{x}+\sum_{\alpha=1}^{n} \int_{0}^{t} \int_{\Omega_{r}}\left|u_{x_{\alpha}}\right|^{p_{\alpha}} d \mathbf{x} d \tau
$$

then 69 can be rewritten as

$$
F_{r+\rho}(t) \leqslant \frac{C_{2}}{\varepsilon \rho}\left(F_{r}(t)+\varepsilon^{p_{s}} \int_{0}^{t} F_{r}(\tau) d \tau\right) .
$$

Let us establish the inequality

$$
F_{R_{0}+l \rho}(t) \leqslant C\left(\frac{2 C_{2}}{\rho}\right)^{l} t^{l / p_{s}}\left\{\prod_{i=0}^{l-1}\left(1+i / p_{s}\right)\right\}^{-1 / p_{s}}\|\varphi\|_{k}^{k}, \quad l=\overline{0, \infty}
$$

by induction in $l$. As the zeroth step of the induction, inequality $(28)$ for each $t>0$ implies inequality $F_{R_{0}}(t) \leqslant C\|\varphi\|_{k}^{k}$. Suppose that (71) holds true for some integer $l \geq 0$. Substituting $\varepsilon=\left[\frac{\left(1+l / p_{s}\right)}{t}\right]^{1 / p_{s}}, r=R_{0}+l \rho$ into 70 and bearing in mind 71 , we obtain

$$
\begin{gathered}
F_{R_{0}+(l+1) \rho}(t) \leqslant C 2^{l}\left(\frac{C_{2}}{\rho}\right)^{l+1} t^{1 / p_{s}}\left\{\prod_{i=0}^{l}\left(1+i / p_{s}\right)\right\}^{-1 / p_{s}}\|\varphi\|_{k}^{k} \times \\
\times\left\{t^{l / p_{s}}+\frac{1+l / p_{s}}{t} \int_{0}^{t} \tau^{l / p_{s}} d \tau\right\}=C\left(\frac{2 C_{2}}{\rho}\right)^{l+1} t^{(l+1) / p_{s}}\left\{\prod_{i=0}^{l}\left(1+i / p_{s}\right)\right\}^{-1 / p_{s}}\|\varphi\|_{k}^{k} .
\end{gathered}
$$

Inequality 71 is proven.

Let $\rho=\left(r-R_{0}\right) / l$. Employing Stirling's approximation, by 71 one can easily get

$$
F_{r}(t) \leqslant C_{3} \exp \left(-\frac{l}{p_{s}} \ln \frac{\left(r-R_{0}\right)^{p_{s}}}{C_{4} t l^{p_{s}-1}}\right)\|\varphi\|_{k}^{k}
$$

Letting $l$ being the integer part of the expression $\left[\frac{\left(r-R_{0}\right)^{p_{s}}}{e C_{4} t}\right]^{1 /\left(p_{s}-1\right)}$, by inequality $\{72$ we obtain

$$
F_{r}(t) \leqslant C_{5} \exp \left(-C_{6}\left[\frac{\left(r-R_{0}\right)^{p_{s}}}{t}\right]^{1 /\left(p_{s}-1\right)}\right)\|\varphi\|_{k}^{k} .
$$

In the case $l=0$ inequality $(73)$ follows from relation (28). Finally, as $r \geq 2 R_{0}$ by $(73)$ we get estimate (7).

Theorem 3 is proven on the basis of the following statement. 
Lemma 4. Suppose the domain is located along the axis $O x_{s}, s \in \overline{2, n}$ and conditions (13), (6) hold true. Then there exists positive numbers $\kappa\left(p_{s}, k\right), \mathcal{M}\left(p_{s}, k\right)$ such that for the constructed bounded solution $u(t, \mathbf{x})$ to problem (1)-(3) for all $t \geq 0, r \geq 2 R_{0}$ the estimate

$$
\|u(t)\|_{k, \Omega_{r}} \leqslant \mathcal{M} \exp \left(-\kappa \int_{1}^{r} \nu^{p_{1} / p_{s}}(\rho) d \rho\right)\|\varphi\|_{k}
$$

holds true.

Proof. Let $\theta(x), x>0$, be an absolute continuous function being one as $x \geq r$, vanishing as $x \leq R_{0}$, being linear as $x \in\left[R_{0}, 2 R_{0}\right]$, and satisfying equation

$$
\theta^{\prime}(x)=\delta \nu^{p_{1} / p_{s}}(x) \theta(x), \quad x \in\left(2 R_{0}, r\right),
$$

(we shall determine the constant $\delta$ later). Solving this equation, we find, in particular, that

$$
\theta^{\prime}(x)=\frac{\theta\left(2 R_{0}\right)}{R_{0}}=\frac{1}{R_{0}} \exp \left(-\delta \int_{2 R_{0}}^{r} \nu^{p_{1} / p_{s}}(\rho) d \rho\right), \quad x \in\left(R_{0}, 2 R_{0}\right) .
$$

For each function $v(\mathbf{x}) \in C_{0}^{\infty}(\Omega)$ the definition of the function $\nu(\rho)$ implies the inequality

$$
\nu(\rho)\|v\|_{p_{1}, \gamma_{\rho}} \leq\left\|v_{x_{1}}\right\|_{p_{1}, \gamma_{\rho}}, \quad \rho>0,
$$

that yields the relations

$$
\int_{2 R_{0}}^{r} \theta^{p_{s}}(\rho) \nu^{p_{1}}(\rho)\|v\|_{p_{1}, \gamma_{\rho}}^{p_{1}} d \rho \leq \int_{2 R_{0}}^{r} \theta^{p_{s}}(\rho)\left\|v_{x_{1}}\right\|_{p_{1}, \gamma_{\rho}}^{p_{1}} d \rho .
$$

Applying (77) for each function $v \in C_{0}^{\infty}(\Omega)$ as $s \in \overline{2, n}$, we deduce

$$
\begin{gathered}
\int_{2 R_{0}}^{r} \nu^{p_{1}}(\rho) \theta^{p_{s}}(\rho)\|v\|_{p_{s}, \gamma_{\rho}}^{p_{s}} d \rho \leq \max _{\Omega}|v(\mathbf{x})|^{p_{s}-p_{1}} \int_{2 R_{0}}^{r} \nu^{p_{1}}(\rho) \theta^{p_{s}}(\rho)\|v\|_{p_{1}, \gamma_{\rho}}^{p_{1}} d \rho \leqslant \\
\leqslant \max _{\Omega}|v(\mathbf{x})|^{p_{s}-p_{1}} \int_{2 R_{0}}^{r} \theta^{p_{s}}(\rho)\left\|v_{x_{1}}\right\|_{p_{1}, \gamma_{\rho}}^{p_{1}} d \rho .
\end{gathered}
$$

We note that inequalities $(1)$ hold true for any bounded function $v \in \stackrel{\circ}{W}_{k, \mathbf{p}}^{1}(\Omega)$ (see [6, Corollary 1]).

In 66 we let $\xi\left(x_{s}\right)=\theta^{p_{s}}\left(x_{s}\right)$ and obtain

$$
\begin{gathered}
\frac{k-1}{k} \int_{\Omega}|u(t, \mathbf{x})|^{k} \theta^{p_{s}}\left(x_{s}\right) d \mathbf{x}+\bar{a} \sum_{\alpha=1}^{n} \int_{D^{t}} \theta^{p_{s}}\left|u_{x_{\alpha}}\right|^{p_{\alpha}} d \mathbf{x} d \tau \leqslant \\
\leqslant \widehat{a} \int_{0}^{t} \int_{\Omega}|u|\left|u_{x_{s}}\right|^{p_{s}-1} p_{s} \theta^{\prime}\left(x_{s}\right) \theta^{p_{s}-1}\left(x_{s}\right) d \mathbf{x} d \tau \equiv \widehat{a} I^{t} .
\end{gathered}
$$

Employing Young inequality, we get

$$
I^{t} \leqslant \varepsilon\left(p_{s}-1\right) \int_{0}^{t} \int_{\Omega}\left|u_{x_{s}}\right|^{p_{s}} \theta^{p_{s}} d \mathbf{x} d \tau+\frac{1}{\varepsilon^{p_{s}-1}} \int_{0}^{t} \int_{\Omega}|u|^{p_{s}}\left(\theta^{\prime}\left(x_{s}\right)\right)^{p_{s}} d \mathbf{x} d \tau .
$$


We choose $\varepsilon=\frac{\bar{a}}{\widehat{a}} \frac{1}{p_{s}-1}$ and mimicking 79, , 80, , we obtain the inequality

$$
\frac{k-1}{k} \int_{\Omega}|u(t, \mathbf{x})|^{k} \theta^{p_{s}}\left(x_{s}\right) d \mathbf{x}+\bar{a} \sum_{\alpha=1, \alpha \neq s}^{n} \int_{D^{t}} \theta^{p_{s}}\left|u_{x_{\alpha}}\right|^{p_{\alpha}} d \mathbf{x} d \tau \leqslant C_{7} \int_{D^{t}}|u|^{p_{s}}\left(\theta^{\prime}\left(x_{s}\right)\right)^{p_{s}} d \mathbf{x} d \tau .
$$

Employing (75), (76), one can easily reduce (81) to

$$
\begin{aligned}
& \frac{k-1}{k} \int_{\Omega}|u(t, \mathbf{x})|^{k} \theta^{p_{s}}\left(x_{s}\right) d \mathbf{x}+\bar{a} \sum_{\alpha=1, \alpha \neq s}^{n} \int_{D^{t}} \theta^{p_{s}}\left|u_{x_{\alpha}}\right|^{p_{\alpha}} d \mathbf{x} d \tau \leqslant \\
& \leqslant C_{7} \frac{1}{R_{0}^{p_{s}}} \exp \left(-\delta p_{s} \int_{2 R_{0}}^{r} \nu^{p_{1} / p_{s}}(\rho) d \rho\right) \int_{0}^{t} \int_{\Omega_{R_{0}}^{2 R_{0}}}|u|^{p_{s}} d \mathbf{x} d \tau+ \\
& +C_{7} \delta^{p_{s}} \int_{0}^{t} \int_{\Omega_{2 R_{0}}^{r}}|u|^{p_{s}} \nu^{p_{1}}\left(x_{s}\right) \theta^{p_{s}}\left(x_{s}\right) d \mathbf{x} d \tau=I_{1}^{t}+I_{2}^{t} .
\end{aligned}
$$

Employing [6, Ineq. (73)] and relation (28), we deduce

$$
I_{1}^{t} \leqslant C_{8} \exp \left(-\delta p_{s} \int_{2 R_{0}}^{r} \nu^{p_{1} / p_{s}}(\rho) d \rho\right) \int_{0}^{t}\left\|u_{x_{s}}\right\|_{p_{s}}^{p_{s}} d \tau \leqslant C_{9} \exp \left(-\delta p_{s} \int_{2 R_{0}}^{r} \nu^{p_{1} / p_{s}}(\rho) d \rho\right)\|\varphi\|_{k}^{k} .
$$

Applying (1), we get

$$
I_{2}^{t} \leqslant C_{10} \delta^{p_{s}} \int_{0}^{t} \int_{\Omega_{2 R_{0}}^{r}}\left|u_{x_{1}}\right|^{p_{1}} \theta^{p_{s}} d \mathbf{x} d \tau
$$

Choosing $\delta=\left(\frac{\bar{a}}{C_{10}}\right)^{1 / p_{s}}$, mimicking 82 - 84 , we obtain

$$
\frac{k-1}{k}\|u(t)\|_{k, \Omega_{r}}^{k}+\bar{a} \sum_{\alpha=2, \alpha \neq s}^{n} \int_{0}^{t}\left\|u_{x_{\alpha}}(t)\right\|_{\Omega_{r}}^{p_{\alpha}} d \tau \leqslant C_{9} \exp \left(-C_{11} \int_{1}^{r} \nu^{p_{1} / p_{s}}(\rho) d \rho\right)\|\varphi\|_{k}^{k} .
$$

Inequality (74) is proven.

Theorem 3 is proven on the basis of estimate (74) by analogy with the proof of Theorem 3 in [6].

\section{BIBLIOGRAPHY}

1. L.M. Kozhevnikova, F.Kh. Mukminov. Estimates of the stabilization rate as $t \rightarrow \infty$ of solutions of the first mixed problem for a quasilinear system of second-order parabolic equations. // Matem. sbornik. 2000. V. 191, No. 2. P. 91-131. [Sb. math. 2000. V. 191, No. 2. P. 235-273.]

2. L.M. Kozhevnikova. Stabilization of a solution of the first mixed problem for a quasi-elliptic evolution equation. // Matem. sbornik. 2005. V. 196, No. 7. P. 67-100. [Sb. math. 2005. V. 196, No. 7. P. 999-1032.]

3. R.Kh. Karimov, L.M. Kozhevnikova. Stabilization of solutions of quasilinear second order parabolic equations in domains with non-compact boundaries // Matem. sbornik. 2010. V. 201, No. 9. P. 3-26. [Sb. math. 2010. V. 201, No. 9. P. 1249-1271.] 
4. S.P. Degtyarev, A.F. Tedeev. $L_{1}-L_{\infty}$ estimates of solutions of the Cauchy problem for an anisotropic degenerate parabolic equation with double non-linearity and growing initial data // Matem. sbornik. 45-66. [Sb. math. 2007. V. 198, No. 5. P. 639-660.]

5. E.R. Andriyanova, F.Kh. Mukminov. The lower estimate of decay rate of solution for doubly nonlinear parabolic equation// Ufimsk. matem. zhurn. 2011. V. 3, No. 3. P. 3-14. [Ufa math. j. 2011. V. 3, No. 3. P. 3-14.]

6. L.M. Kozhevnikova, A.A. Leontiev. Estimates of solutions of anisotropic doubly nonlinear parabolic equation // Ufimsk. matem. zhurn. 2011. V. 3, No. 4. P. 64-85. [Ufa math. j. 2011. V. 3, No. 4. P. 62-83.]

7. A.F. Tedeev. Stabilization of the solutions of initial-boundary value problems for quasilinear parabolic equations // Ukr. matem. zhurn. 1992. V. 44, No. 10. P. 1441-1450. [Ukr. math. j. 1992. V. 44, No. 10. P. 1325-1334.]

8. N. Alikakos, R. Rostamian Gradient estimates for degenerate diffusion equation. II // Proc. Roy. Soc. Edinburgh. V. 91, 3-4. 1981/1982. P. 335-346.

9. V.A. Trenogin. Functional analysis. Nauka, Moscow. 1980. (in Russian).

10. J.L. Lions. Quelques méthodes de résolution des problèmes aux limites non linéaires. Etudes mathematiques. Dunod, Gauthier-Villars, Paris. 1969.

11. G. Sansone. Equazioni differenziali nel campo reale. II. Consiglio nazionale delle Ricerche. Monografie di Matematica applicata. N. Zanichelli Editore. Bologna. 1949.

Larisa Mikhailovna Kozhevnikova,

Sterlitamak Branch of Bashkir State University,

Lenin av., 37,

453103, Sterlitamak, Russia

E-mail: kosul@mail.ru

Alexei Alexandrovich Leontiev,

Sterlitamak Branch of Bashkir State University

Lenin av., 37,

453103, Sterlitamak, Russia 